\title{
Blood and urinary metabolomic evidence validating traditional Chinese medicine diagnostic classification of major depressive disorder
}

\author{
Lan-Ying Liu' ${ }^{1}$, Hong-Jian Zhang ${ }^{2}$, Li-Yuan Luo' ${ }^{1}$ Jin-Bao Pu², Wei-Qing Liang ${ }^{2}$, Chun-Qin Zhu' ${ }^{1}$, Ya-Ping Li ${ }^{3}$,
} Pei-Rong Wang ${ }^{1}$, Yuan-Yuan Zhang ${ }^{1}$, Chun-Yu Yang ${ }^{1}$ and Zhang-Jin Zhang ${ }^{4^{*}}$

\begin{abstract}
Background: Major depressive disorder (MDD) is a highly heterogeneous disease. Further classification may characterize its heterogeneity. The purpose of this study was to examine whether metabolomic variables could differentiate traditional Chinese medicine (TCM) diagnostic subtypes of MDD.

Methods: Fifty medication-free patients who were experiencing a recurrent depressive episode were classified into Liver Qi Stagnation ( $(L Q S, n=30)$ and Heart and Spleen Deficiency (HSD, $n=20)$ subtypes according to TCM diagnosis. Healthy volunteers $(n=28)$ were included as controls. Gas chromatography-mass spectrometry (GC-MS) was used to examine serum and urinary metabolomic profiles.

Results: Twenty-eight metabolites were identified for good separations between TCM subtypes and healthy controls in serum samples. Both TCM subtypes had similar profiles in proteinogenic branched-chain amino acids (BCAAs) (valine, leucine, and isoleucine) and energy metabolism-related metabolites that were differentiated from healthy controls. The LQS subtype additionally differed from healthy controls in multiple amino acid metabolites that are involved in biosynthesis of monoamine and amino acid neurotransmitters, including phenylalanine, 3-hydroxybutric acid, o-tyrosine, glycine, L-tryptophan, and N-acetyl--aspartic acid. Threonic acid, methionine, stearic acid, and isobutyric acid are differentially associated with the two subtypes.
\end{abstract}

Conclusions: While both TCM subtypes are associated with aberrant BCAA and energy metabolism, the LQS subtype may represent an MDD subpopulation characterized by abnormalities in the biosynthesis of monoamine and amino acid neurotransmitters and closer associations with stress-related pathophysiology. The metabolites differentially associated with the two subtypes are promising biomarkers for predicting TCM subtype-specific antidepressant response [registered at http://www.clinicaltrials.gov (NCT02346682) on January 27, 2015].

Keywords: Major depressive disorder, Traditional Chinese medicine, Classification, Metabolomics

\footnotetext{
${ }^{*}$ Correspondence: zhangzj@hku.hk

${ }^{4}$ School of Chinese Medicine, LKS Faculty of Medicine, The University

of Hong Kong, 10 Sassoon Road, Pokfulam, Hong Kong, China

Full list of author information is available at the end of the article
} 


\section{Background}

Major depressive disorder (MDD) is a highly heterogeneous mental illness with a wide range of clinical manifestations and multi-system etiopathogenesis. The core symptoms of low mood and loss of interest and pleasure are often accompanied by somatic and other psychiatric symptoms, such as pain, sleep disturbance, anxiety, and neurocognitive dysfunction [1]. Although a great attempt has been made to characterize its heterogeneity by subdividing MDD based on its clinical features, severity, and polygenic features, convincing evidence for the existence of depressive symptom dimensions and symptomatic subtypes is lacking, mainly due to symptomatic diversity and the absence of patterns [2-5]. Therefore, a novel classification system that could improve clinical applicability and more precisely identify pathological heterogeneity of MDD is highly desirable.

As an ancient medical practice, traditional Chinese medicine (TCM) has an established distinct diagnostic system. One key approach to diagnosis is the differentiation of etiopathological pattern which is generally defined by a comprehensive analysis of clinical symptoms and signs collected through inspection, auscultation, olfaction, interrogation, and palpation of the pulses [6]. Over the past decade, several large-scale studies have revealed 12 different TCM patterns of MDD on the basis of the modern psychiatric diagnostic instruments and analysis tools, such as latent tree model analysis [7-12]. Despite disagreements on some patterns, a consensus on clinical diagnostic criteria for the two most common and opposing patterns, Liver Qi Stagnation (LQS) and Heart and Spleen Deficiency (HSD), which account for approximately $2 / 3$ of depressed patients, have been reached as shown in Table $1[7,9,10]$.

Differential profiles of functional brain connectivity has been observed between "deficiency" and "excessive" patterns of MDD [13]. One recent study has further revealed differences between patients with liver-depression and spleen-deficiency syndrome and healthy volunteers in multiple serum metabolic pathways associated with amino acid and energy metabolism [14]. On the other hand, aberrant amino acid metabolism that is involved in protein synthesis, energy metabolism, monoamine and amino acid neurotransmitter biosynthesis has been suggested in the pathogenesis of MDD and antidepressant response [15]. Differential metabolomic profiles are

Table 1 Clinical manifestations and diagnostic criteria of TCM-based subtypes of MDD

\begin{tabular}{|c|c|c|}
\hline & Liver Qi Stagnation (LQS) & Heart and Spleen Deficiency (HSD) \\
\hline Mood symptom characters & $\begin{array}{l}\text { Depressed mood with frustration, nervousness, and/or } \\
\text { irritability }\end{array}$ & $\begin{array}{l}\text { Depressed mood with excessive pensiveness, suspicion, } \\
\text { and/or timorousness }\end{array}$ \\
\hline Somatic symptoms & $\begin{array}{l}\text { A. Frequently sighing }(0=\text { absent, } 1=\text { slight, } 2=\text { mild, } \\
3=\text { moderate, } 4=\text { severe }) \\
\text { B. Chest distension and/or hypochondriac pain }(0=\text { absent, } \\
1=\text { slight, } 2=\text { mild, } 3=\text { moderate, } 4=\text { severe }) \\
\text { C. Abdominal bloating }(0=\text { absent, } 1=\text { slight, } 2=\text { mild, } \\
3=\text { moderate, } 4=\text { severe }) \\
\text { D. Decreased appetite }(0=\text { absent, } 1=\text { mild, } 2=\text { moderate, } \\
3=\text { severe }) \\
\text { E. Loose stool }(0=\text { absent, } 1=\text { mild, } 2=\text { moderate, } \\
3=\text { severe) } \\
\text { F. Breast tenderness }(0=\text { absent, } 1=\text { slight, } 2=\text { mild, } \\
3=\text { moderate, } 4=\text { severe }) \\
\text { G. Irregular menstruation }(0=\text { normal, } 1=\text { seldom, } \\
2=\text { sometimes, } 3=\text { frequent })^{\text {a }} \\
\text { H. Menstrual pain }(0=\text { absent, } 1=\text { mild, } 2=\text { moderate, } \\
3=\text { severe })^{a}\end{array}$ & $\begin{array}{l}\text { A. Palpitation }(0=\text { normal, } 1=\text { seldom, } 2=\text { sometimes, } \\
3=\text { most times, } 4=\text { all times }) \\
\text { B. Forgetfulness }(0=\text { absent, } 1=\text { slight, } 2=\text { mild, } 3=\text { moder- } \\
\text { ate, } 4=\text { severe }) \\
\text { C. Insomnia or dream-disturbed sleep ( } 0=\text { absent, } 1=\text { some- } \\
\text { times, } 3=\text { most times }) \\
\text { D. Decreased appetite }(0=\text { absent, } 1=\text { slight, } 2=\text { mild, } \\
3=\text { moderate, } 4=\text { severe) } \\
\text { E. Abdominal fullness }(0=\text { absent, } 1=\text { sometimes, } 3=\text { most } \\
\text { times) } \\
\text { F. Loose stool or dysfunctional diarrhea ( } 0=\text { absent, } \\
1=\text { slight, } 2=\text { mild, } 3=\text { moderate, } 4=\text { severe) } \\
\text { G. Pale and sallow complexion }(0=\text { normal, } 1=\text { slightly } \\
\text { apparent, } 2=\text { mildly apparent; } 3=\text { moderately apparent, } \\
4=\text { very apparent) } \\
\text { H. Tiredness }(0=\text { absent, } 1=\text { mild, } 2=\text { moderate, } 3=\text { severe) }\end{array}$ \\
\hline Tongue and pulse & $\begin{array}{l}\text { I. Red tongue body with thin and white coating }(0=\text { nor- } \\
\text { mal, } 1=\text { mildly apparent, } 2=\text { moderately apparent, } \\
3=\text { very apparent) } \\
\text { J. Wiry pulse }(0=\text { normal, } 1=\text { slightly apparent, } 2=\text { mildly } \\
\text { apparent; } 3=\text { moderately apparent, } 4=\text { very apparent) }\end{array}$ & $\begin{array}{l}\text { I. Pale and tender or watery tongue body with white coating } \\
(0=\text { normal, } 1=\text { slightly apparent, } 2=\text { mildly apparent; } \\
3=\text { moderately apparent, } 4=\text { very apparent }) \\
\text { J. Weak and thin pulse }(0=\text { normal, } 1=\text { slightly apparent, } \\
2=\text { mildly apparent; } 3=\text { moderately apparent, } 4=\text { very } \\
\text { apparent) }\end{array}$ \\
\hline Diagnostic criteria $^{b}$ & $\begin{array}{l}\text { [1]. Must have } A, B, I \text {, and J; at least one of } D \text { and } E \text {; and at } \\
\text { least one of } F, G \text {, and } H \text { for women } \\
\text { [2]. Total score of physical symptoms and tongue and pulse } \\
\text { signs is not less than } 10\end{array}$ & $\begin{array}{l}\text { [1]. Must have at least two of } \mathrm{A}, \mathrm{B} \text { and } \mathrm{C} \text {; at least two of } \mathrm{D}, \mathrm{E} \\
\text { and } \mathrm{F} \text {; at least one of } \mathrm{G} \text { and } \mathrm{H} ; \mathrm{I} \text { and } \mathrm{J} \\
\text { [2]. Total score of physical symptoms and tongue and pulse } \\
\text { signs is not less than } 10\end{array}$ \\
\hline
\end{tabular}

The diagnostic criteria are modified based on Ref. $[7,9,10]$

a $F, G$, and $H$ items are only applied for women

b Those who fail to meet either LQS or HSD subtype are classified as Other Subtypes 
also associated with the severity of MDD and whether the patients had early life stress and suicidal ideation [16-20]. These studies have led to the hypothesis that certain metabolic pathways particularly associated with protein synthesis, energy metabolism, and biosynthesis of monoamine and amino acid neurotransmitters could serve valid biomarkers for differentiating TCM diagnostic subtypes of MDD.

To test this hypothesis, this study was designed to examine whether blood and urinary metabolomic profiles were different between the LQS and HSD subtypes of MDD with the inclusion of healthy volunteers as controls.

\section{Materials and methods Setting and participants}

This study was conducted at Tongde Hospital of Zhejiang Province in Hangzhou, China between April 2015 and March 2017. The study protocol was approved by the Medical Ethical Committee [equivalent to the Institutional Review Board (IRB)] of Tongde Hospital of Zhejiang Province on March 11, 2014 (The approved document is attached in Additional file 1) and registered at http://www.clinicaltrials.gov (NCT02346682) on January 27, 2015 (https://clinicaltrials.gov/ct2/show/ NCT02346682). All participants gave voluntary, written, informed consent before entering the study. We reported this study according to the Minimum Standards of Reporting Checklist (see Additional file 2).

Participants were sought from out-inpatients. Subjects were eligible for this study if they: (a) were aged 18-65 years; (b) were currently experiencing a recurrent, moderate or severe depressive episode according to the Diagnostic and Statistical Manual of Mental Disorders, Fifth Edition (DSM-5), as evidenced by a score of at least
21 on the 24-item Hamilton Rating Scale for Depression (HAMD-24) [21]; (c) met the diagnostic criteria of the LQS or HSD subtype as defined in Table 1 with representative tongue manifestations as shown in Fig. 1; and (d) had received no treatment with antidepressants and other psychotropic drugs in the previous 3 months. Tongue diagnosis is a critical diagnostic approach in discriminating different TCM patterns according to manifestations of the tongue coating and body [22].

Patients were excluded from this study if they: (a) were experiencing their first episode of depression; (b) had serious comorbid cardiac, hepatic, or renal conditions; (c) had a history of brain injury or surgery; (d) had a history of manic, hypomanic, or mixed episodes; (e) had received investigational drug treatment within the previous 6 months; (f) had experienced alcohol or drug abuse within the previous 12 months; or (g) were pregnant or breastfeeding.

A group of healthy volunteers who had no personal or family history of significant mental and physical illness was recruited from Tongde Hospital and local community to serve as controls.

\section{Screening, assessment, and TCM diagnosis}

The screening of patients and healthy volunteers was done by a psychiatrist and a TCM practitioner. The severity of the depression symptoms of the patients and healthy controls was assessed using HAMD-24 [21]. The assessment was conducted by a trained rater (L.Y.L., C.Q.Z.). TCM subtypes diagnoses were made by at least two senior TCM practitioners (Y.P.L., C.Q.Z.) and a third TCM practitioner (L.Y.L.) was involved in the diagnosis process if the first two practitioners could not reach an agreement.

To ensure consistency of assessment and diagnosis, a manual was provided and a training workshop was

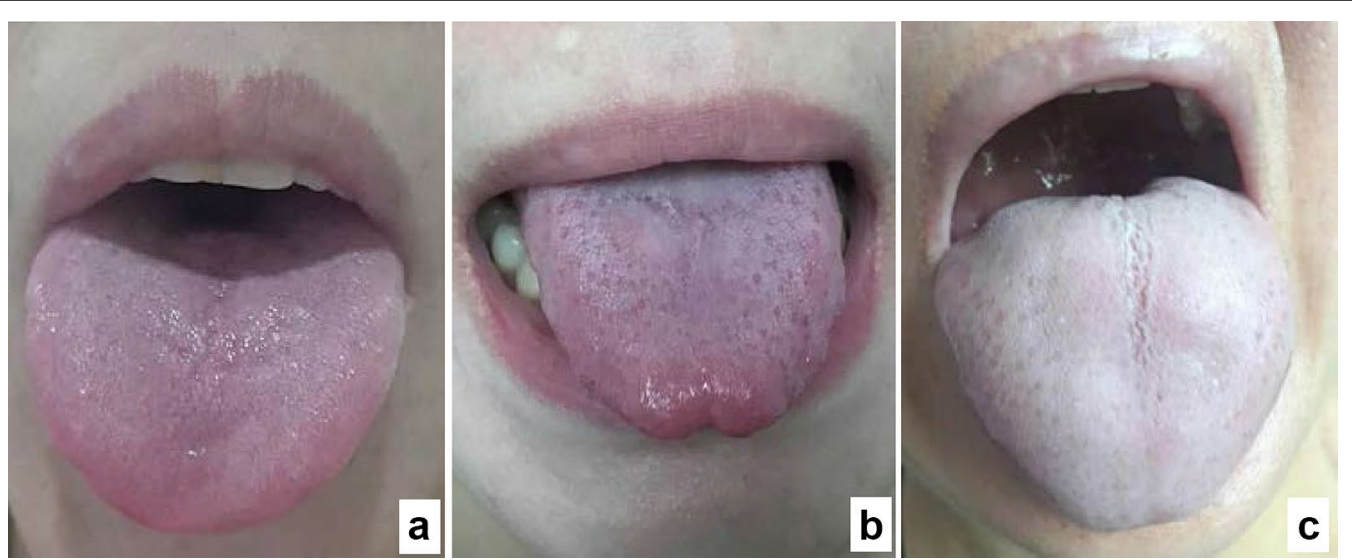

Fig. 1 Representative tongue pictures taken from a healthy volunteer (a), depressed patients with Liver Qi Stagnation (b) and Heart Spleen Deficiency (c) subtypes 
carried out on videotaped patients with different TCM subtypes before the study was initiated. An inter-rater reliability coefficient of $>0.85$ was achieved after the completion of the training workshop.

\section{Collection and preparation of serum and urine samples}

Blood and urine sample for each were collected within 2 days after the completion of clinical assessment. Following the sample collection, patients immediately received antidepressant and other psychotropic treatment at psychiatrists' discretion based on their clinical condition, with routine subsequent monitoring.

In the morning (09:00-10:00) after an overnight fasting, $10 \mathrm{ml}$ of midstream urine were collected; $15 \mathrm{ml}$ of blood were drawn and sera were immediately separated. Samples were further prepared for metabolomic measurement as described previously [17, 23]. Briefly, $500 \mu \mathrm{l}$ of urine or sera was mixed with $50 \mu \mathrm{l}$ heptanoic acid and $10 \mathrm{mg} / \mathrm{ml}$ para-chlorophenylalanine as the internal standard solution. $500 \mu \mathrm{l}$ methanol was added to precipitate protein, mixed for $5 \mathrm{~min}$, and then centrifuged at $12,000 \mathrm{rpm} / \mathrm{min}$ for $15 \mathrm{~min}$ at $4{ }^{\circ} \mathrm{C}$. A $500-\mu \mathrm{l}$ aliquot of supernatant was transferred to a clean Eppendorf tube and dried under a low temperature vacuum drier. The residue was derivatized by adding $100 \mu \mathrm{l}$ methoxyamine hydrochloride $(15 \mathrm{mg} / \mathrm{ml}$ in pyridine $)$ at $30{ }^{\circ} \mathrm{C}$ for $90 \mathrm{~min}$. For each sample, $100 \mu \mathrm{l}$ BSTFA (1\% TMCS) was added and the mixture was heated at $70{ }^{\circ} \mathrm{C}$ for $60 \mathrm{~min}$. The derivative was cooled and filtered in $0.45 \mu \mathrm{m}$ membrane prior to GC-MS analysis. Quality control samples and the reference standard were processed as done for experimental samples.

\section{GC-MS acquisition}

One $\mu \mathrm{l}$ aliquot of derivatized sample was injected into a Varian 450-GC/240-MS equipped with $19091 \mathrm{~N}-113$ capillary column $(30 \mathrm{~m} \times 0.32 \mathrm{~mm} \times 0.25 \mu \mathrm{m}$, Agilent $\mathrm{J} \& \mathrm{~W}$ Scientific, USA) at a split ratio of 10:1. Helium was used as the carrier gas with a constant flow rate of $1 \mathrm{ml} / \mathrm{min}$. The initial temperature was set at $70{ }^{\circ} \mathrm{C}$ for $4 \mathrm{~min}$, elevated to $300^{\circ} \mathrm{C}$ at a rate of $8^{\circ} \mathrm{C} / \mathrm{min}$, and then maintained for $3 \mathrm{~min}$. Temperature for the injector, transfer line, and ion source was set at $280^{\circ} \mathrm{C}, 250^{\circ} \mathrm{C}$, and $220^{\circ} \mathrm{C}$, respectively. The mass range $(50-800 \mathrm{~m} / \mathrm{z})$ in a full-scan mode for electron impact ionization $(1.0 \mathrm{kV})$ was applied. The solvent delay time was set to $6 \mathrm{~min}$.

\section{Statistical analysis}

There were no studies detecting metabolomic effects in TCM subtypes of MDD. Sample size estimation was based on one previous study that has revealed differential metabolomic profiles between MDD patients with and without early life stress [17]. As the LQS and HSD subtypes are appear to be differentially associated with stress-related (reactive) and endogenous (melancholic) depression [2, 9], we assumed that metabolomic differences between the two TCM subtypes was similar to that of MDD patients with and without early life stress [17]. The study has shown an averaged $36 \%$ difference in plasma level of major metabolites between MDD patients with and without early life stress with an averaged standard deviation of $41 \%$ [17]. A sample size of 22 each group would be sufficient to yield an $80 \%$ power at a statistical level of 0.05 .

For baseline data, one-way analysis of variance (ANOVA) was used to detect differences in continuous variables among healthy controls and the two TCM subtypes. Student's $t$-test was used to detect differences in continuous variables between the two TCM subtypes. Categorical baseline variables were analyzed using Chi square $\left(x^{2}\right)$ test.

For metabolomic data, the pretreatment process was performed, including novel nonlinear retention time alignment, baseline filtration, peak identification, matching, and integration. The resulting data matrix consisting of variables, sample code, and peak area was further processed using Microsoft Excel program. The original spectral data obtained from GC-MS spectroscopy were scaled to unit variance $(z)$, which was calculated from the formula $z=(x-y) / s$, where $x, y$, and $s$ represent the level of the particular metabolite in one subject, the mean level and the standard deviation of this metabolite across all subjects, respectively.

Nonparametric Mann-Whitney $U$ test was used to detect significantly differential metabolites. All metabolites were determined by standard samples and/or a similarity of $>70 \%$ that was obtained by comparing with the mass spectral database of the US National Institute for Standards and Technology (NIST).

Metabolites were determined using variable importance in the projection (VIP) which value was defined as $>1$ and $t$-test was set at a level of $95 \%$. The principal component analysis (PCA) was used to discriminate metabolic patterns among the three groups. The orthogonal projection to latent structures-discriminant analysis (OPLS-DA) model was further constructed to identify meaningful metabolites that could differentiate between the three groups using SIMCA-P 13.0 program. A regression method was applied to establish the optimal discriminant model with controlling gender as a confounding factor. The quality of the model was tested with cross-validation and $R^{2} X, R^{2} Y$, and $Q^{2}$ values were obtained. OPLS-DA models which all $R^{2} X, R^{2} Y$, and $Q^{2}$ values were $\geq 0.5$ were acceptable. Hierarchical clustering patterns were further established with heatmap analysis using R i386 3.3.0 software for visual verification of 
OPLS-DA models. The impact pathway was determined using MetaboAnalyst 3.0 (http://www.metaboanalyst. ca), a web-based tool for pathway analysis, with the criteria as $p$-value $<0.05$, false discovery rate $(\mathrm{FDR})<0.05$, and impact value $>0$. Visualization of metabolomic correlation network was drawn with CytoScape 3.3.0. The receiver operating characteristic (ROC) curve analysis was conducted to determine the optimal metabolite combination patterns that could well dichotomize the subtypes and healthy controls at acceptable sensitivity and specificity (defined as greater than $80 \%$ for both).

Statistical analysis was conducted using SPSS 18.0 (SPSS Inc., Chicago, IL, USA) and, unless otherwise indicated, significance level was set at a two-tailed $P<0.05$.

\section{Results}

\section{Participant characteristics}

Flowchart of screening and recruitment is shown in Fig. 2. Of 243 depressed patients screened, 189 met the diagnostic criteria of LQS $(n=102)$ and SHD $(n=87)$ subtype; $27.0 \%$ (51/189) rejected to participate the study; 30 LQS subtypes and 20 SHD subtypes who met the inclusion criteria but did not meet any exclusion criteria were recruited. Twenty-eight healthy volunteers were recruited to serve as controls. The proportion of female participants of the LQS subtype was significantly higher than that of the HSD subtype $\left(x^{2}=6.696, p=0.035\right)$ (Table 2). Other demographic and clinical variables were not different among the three groups.

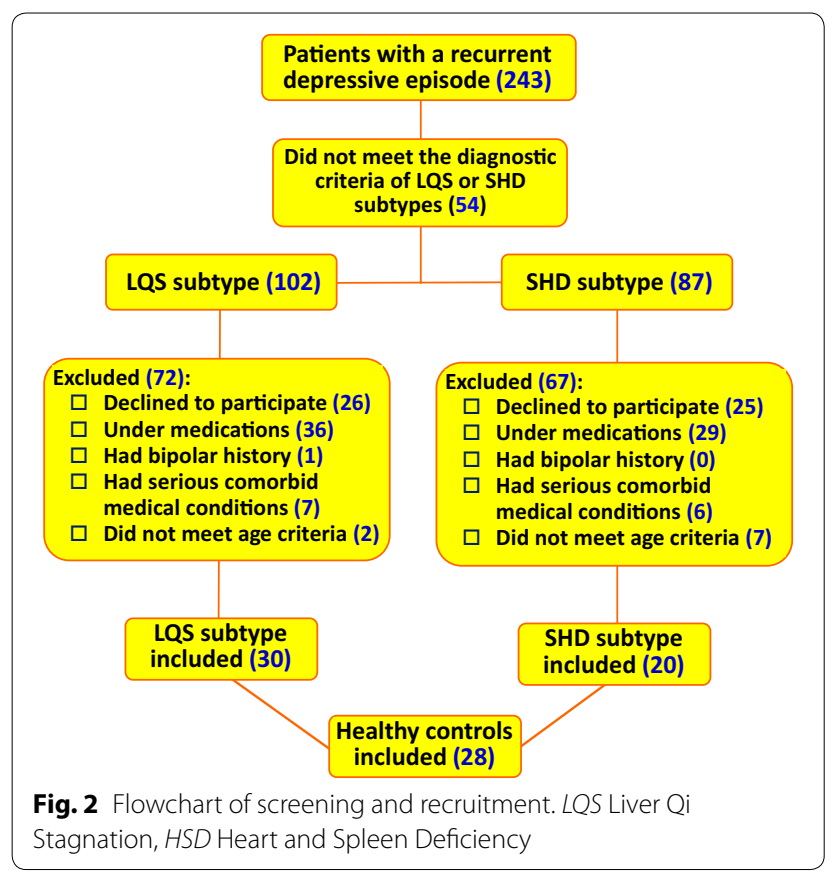

\section{Clustering patterns of differential metabolite profiles}

A total of 28 metabolites were identified to well differentiate the two TCM subtypes from healthy controls (Table 3); 8 of them were present in both serum and urine samples and/or in the two subtypes. The OPLS-DA models revealed that serum metabolite profiles of both subtypes were clearly separated from healthy controls with $R^{2} X$ values of $\geq 0.842, R^{2} Y$ values of $\geq 0.670$, and $Q^{2}$ values of $\geq 0.502$ (Fig. 3a, b). Urinary metabolite profiles of the two subtypes were not well separated from healthy controls, with considerable overlaps and low $R^{2} Y$ and $Q^{2}$ values (Fig. 2d, e). The two subtypes showed large overlaps in serum and urinary metabolite profiles with low $R^{2} Y$ and $Q^{2}$ values (Fig. 2c, f). Hierarchical clustering analysis displayed remarkable differences in mentalism between the three groups, visually validating OPLS-DA models in serum and urine samples (Figs. 4 and 5).

\section{Differential metabolites of TCM subtypes Between LQS subtype and healthy controls}

24 metabolites were valid in discriminating the LQS subtype from healthy controls (Fig. 6, Table 3). In serum samples, levels of L-valine, L-phenylalanine, L-lysine, L-proline, L-leucine, and isobutyric acid were markedly higher than those of healthy controls and positively correlated with the LQS subtype. L-lactic acid level of the LQS subtype was greater than that of healthy subjects with negative correlation with the subtype. Levels of glycine and threonic acid were significantly lower than those of healthy controls and negatively correlated with the LQS subtype.

In urine samples, levels of L-sorbose, diacetyl, $N$-acetylD-glucosamine, L-methionine, 3-hydroxybutyric acid, pyruvic acid, stearic acid, trans-aconitic acid, glycine, $\mathrm{N}$-acetyl-L-aspartic acid, and threonic acid were significantly higher than those of healthy subjects and positively correlated with the LQS subtype. Levels of L-tryptophan, $\alpha$-lactose, L-lactic acid, palmitic acid, indoxyl sulfate, and o-tyrosine were significantly greater than those of healthy subjects but negatively correlated with the LQS subtype. Levels of $p$-hydroxyphenylacetic acid and citric acid were significantly lower higher than those of healthy subjects and negatively correlated with the LQS subtype.

\section{Between HSD subtype and healthy controls}

13 metabolites were identified to be effective in distinguishing the HSD subtype from healthy controls (Fig. 6, Table 3). In serum samples, levels of L-valine, L-leucine, and L-lysine were remarkably higher than those of healthy subjects and positively correlated with the HSD subtype. Levels of L-threonine, D-chiro-Inositol, 
Table 2 Demographic and clinical characteristics of participants

\begin{tabular}{|c|c|c|c|c|}
\hline Variables & $\begin{array}{l}\text { Healthy controls } \\
(n=28)\end{array}$ & LQS subtype $(n=30)$ & HSD subtype $^{a}(n=20)$ & $p$ value ${ }^{b, c}$ \\
\hline Female, $\mathrm{n}(\%)^{\mathrm{b}}$ & $17(60.7)$ & $25(83.3)$ & $10(50.0)$ & 0.035 \\
\hline Age $(y)^{c}$ & $34.1 \pm 8.1$ & $38.1 \pm 13.4$ & $41.5 \pm 13.7$ & 0.107 \\
\hline Educational level, $\mathrm{n}(\%)^{\mathrm{b}}$ & & & & 0.331 \\
\hline Primary & $2(7.1)$ & $6(20.0)$ & $5(25.0)$ & \\
\hline Middle & $5(17.9)$ & $4(13.3)$ & $5(25.0)$ & \\
\hline High $^{d}$ & $21(75.0)$ & $20(66.7)$ & $10(50.0)$ & \\
\hline Marital status, $\mathrm{n}(\%)^{\mathrm{b}}$ & & & & 0.734 \\
\hline Married & $23(82.1)$ & $26(86.7)$ & $18(90.0)$ & \\
\hline Single/divorced/widowed & $5(17.9)$ & $4(13.3)$ & $2(10.0)$ & \\
\hline Income level, n (\%) & & & & 0.775 \\
\hline Low & $4(14.3)$ & $7(23.3)$ & $3(15.0)$ & \\
\hline Middle & $17(60.7)$ & $18(60.0)$ & $14(70.0)$ & \\
\hline High & $7(25.0)$ & $5(16.7)$ & $3(15.0)$ & \\
\hline $\begin{array}{l}\text { Family history with severe mental diseases, } \\
\mathrm{n}(\%)^{\mathrm{b}, \mathrm{f}}\end{array}$ & $3(10.7)$ & $4(13.3)$ & 0 & 0.250 \\
\hline Comorbid conditions, $\mathrm{n}(\%)^{\mathrm{b}, \mathrm{g}}$ & & $3(10)$ & $4(20)$ & 0.560 \\
\hline Medication history, n (\%) ${ }^{\mathrm{b}, \mathrm{h}}$ & & & & 0.803 \\
\hline SSRIS/SNRIs ${ }^{\mathrm{a}}$ & & $25(83.3)$ & $18(90.0)$ & \\
\hline Antipsychotics & & $5(16.7)$ & $2(10.0)$ & \\
\hline Previous depressive episode, $\mathrm{n}(\%)^{\mathrm{b}}$ & & & & 0.684 \\
\hline Single & & $18(60.0)$ & $10(50.0)$ & \\
\hline Multiple & & $12(40.0)$ & $10(50.0)$ & \\
\hline Duration of the illness (months) ${ }^{c, i}$ & & $39.5 \pm 60.9$ & $33.6 \pm 47.8$ & 0.716 \\
\hline HAMD-24 score ${ }^{a, c}$ & & $32.5 \pm 8.5$ & $30.1 \pm 6.5$ & 0.286 \\
\hline
\end{tabular}

a LQS Liver Qi Stagnation, HSD Heart and Spleen Deficiency, SSR/s selective serotonin reuptake inhibitors, SNRIs serotonin-norepinephrine reuptake inhibitors, HAMD24 24-item Hamilton Rating Scale for Depression

b Categorical data was analyzed using Chi square $\left(x^{2}\right)$ test

c Continuous data are expressed mean \pm SD and analyzed using one-way analysis of variance (ANOVA) for the three groups and Student $t$-test for LQS and HSD subtypes

d High levels included high school, college, and postgraduate education

e Compared to average local household incomes

f Severe mental diseases mainly included schizophrenia, bipolar disorders, and severe depression

$g$ Most comorbid conditions were cardiovascular and diabetic diseases

h SSRIs mainly included paroxetine, sertraline, citalopram, and fluoxetine. SNRIs mainly include venlafaxine and duloxetine. Antipsychotics mainly include quetiapine, olanzapine, and risperidone. All medications were taken earlier than 3 months ago at screening

i Duration of the illness was calculated from the first episode of depression

L-methionine, and L-lactic acid were significantly lower than those of healthy subjects and negatively correlated with the HSD subtype.

In urine samples, levels of L-Isoleucine, isobutyric acid, threonic acid, and $\mathrm{N}$-acetyl-D-glucosamine were considerably higher than those of healthy subjects and positively correlated with the HSD subtype. Level of stearic acid was also significantly higher than that of healthy controls but negatively correlated with the HSD subtype. Level of $\alpha$-lactose was significantly lower than that of healthy controls and negatively correlated with the HSD subtype.

\section{Between LQS and HSD subtypes}

10 metabolites showed correlations with both subtypes (Fig. 6). They are urine $\alpha$-lactose, $N$-acetyl-D-glucosamine, and stearic acid; serum L-lactic acid, L-lysine, L-valine, and L-leucine; and isobutyric acid, threonic acid, and L-methionine in both serum and urine samples. Most metabolite correlates with the two subtypes were consistent. Serum threonic acid correlate was negative with the LQS subtype but positive with the HSD subtype. Urine stearic acid correlate was positive with the LQS subtype but negative with the HSD subtype. L-Methionine correlate was positive with the LQS subtype in urine 
Table 3 Differential metabolites identified from MDD patients with TCM subtypes and healthy controls ${ }^{\text {a }}$

\begin{tabular}{|c|c|c|c|c|c|c|}
\hline Group samples & Metabolites & Formula & $r^{\mathbf{b}}$ & $p$-value ${ }^{c}$ & $\mathrm{FC}(\mathrm{MDD} / \mathrm{HC})^{\mathrm{a}}$ & Pathway \\
\hline \multicolumn{7}{|l|}{ Serum: LQS vs. $H C^{a}$} \\
\hline $1^{d}$ & L-Valine & $\mathrm{C}_{5} \mathrm{H}_{11} \mathrm{NO}_{2}$ & 0.236 & 0.009 & 0.930 & Aminoacyl-tRNA biosynthesis \\
\hline $2^{d}$ & L-Phenylalanine & $\mathrm{C}_{9} \mathrm{H}_{11} \mathrm{NO}_{2}$ & 0.199 & 0.237 & 0.350 & Aminoacyl-tRNA biosynthesis \\
\hline $3^{d}$ & L-Lysine & $\mathrm{C}_{6} \mathrm{H}_{14} \mathrm{~N}_{2} \mathrm{O}_{2}$ & 0.150 & 0.761 & 0.459 & Aminoacyl-tRNA biosynthesis \\
\hline 4 & L-Proline & $\mathrm{C}_{5} \mathrm{H}_{9} \mathrm{NO}_{2}$ & 0.143 & 0.447 & 0.182 & Aminoacyl-tRNA biosynthesis \\
\hline $5^{d}$ & L-Leucine & $\mathrm{C}_{6} \mathrm{H}_{13} \mathrm{NO}_{2}$ & 0.124 & 0.000 & 1.047 & Aminoacyl-tRNA biosynthesis \\
\hline $6^{e}$ & Isobutyric acid & $\mathrm{C}_{4} \mathrm{H}_{8} \mathrm{O}_{2}$ & 0.103 & 0.565 & 0.702 & Protein digestion and absorption \\
\hline 7 & L-Lactic acid & $\mathrm{C}_{3} \mathrm{H}_{6} \mathrm{O}_{3}$ & -0.147 & 0.370 & 0.013 & Propanoate metabolism \\
\hline 8 & Glycine & $\mathrm{C}_{2} \mathrm{H}_{5} \mathrm{NO}_{2}$ & -0.151 & 0.279 & -0.954 & Aminoacyl-tRNA biosynthesis \\
\hline 9 & Threonic acid & $\mathrm{C}_{4} \mathrm{H}_{8} \mathrm{O}_{5}$ & -0.175 & 0.250 & -1.080 & Ascorbate and aldarate metabolism \\
\hline \multicolumn{7}{|l|}{ Urine: LQS vs. $H C^{\mathrm{a}}$} \\
\hline 1 & L-Sorbose & $\mathrm{C}_{6} \mathrm{H}_{12} \mathrm{O}_{6}$ & 0.149 & 0.624 & 0.661 & Unknown \\
\hline 2 & Diacetyl & $\mathrm{C}_{4} \mathrm{H}_{6} \mathrm{O}_{2}$ & 0.147 & 0.120 & 0.825 & Butanoate metabolism \\
\hline 3 & N-Acetyl-D-glucosamine & $\mathrm{C}_{8} \mathrm{H}_{15} \mathrm{NO}_{6}$ & 0.146 & 0.748 & 0.304 & Amino sugar and nucleotide sugar metabolism \\
\hline $4^{d}$ & L-Methionine & $\mathrm{C}_{5} \mathrm{H}_{11} \mathrm{NO}_{2} \mathrm{~S}$ & 0.144 & 0.417 & 0.546 & Cysteine and methionine metabolism \\
\hline $5^{d}$ & 3-Hydroxybutyric acid & $\mathrm{C}_{4} \mathrm{H}_{8} \mathrm{O}_{3}$ & 0.139 & 0.088 & 0.819 & Butanoate metabolism \\
\hline $6^{d}$ & Pyruvic acid & $\mathrm{C}_{3} \mathrm{H}_{4} \mathrm{O}_{3}$ & 0.125 & 0.116 & 1.085 & Citrate cycle (TCA cycle) \\
\hline $7^{d}$ & Stearic acid & $\mathrm{C}_{18} \mathrm{H}_{36} \mathrm{O}_{2}$ & 0.117 & 0.327 & 0.490 & Fatty acid biosynthesis \\
\hline 8 & trans-Aconitic acid & $\mathrm{C}_{6} \mathrm{H}_{6} \mathrm{O}_{6}$ & 0.115 & 0.648 & 0.444 & C5-Branched dibasic acid metabolism \\
\hline $9^{d}$ & Glycine & $\mathrm{C}_{2} \mathrm{H}_{5} \mathrm{NO}_{2}$ & 0.111 & 0.685 & 0.441 & Aminoacyl-tRNA biosynthesis \\
\hline $10^{\mathrm{d}}$ & N-Acetyl-L-aspartic acid & $\mathrm{C}_{6} \mathrm{H}_{9} \mathrm{NO}_{5}$ & 0.107 & 0.176 & 0.680 & Alanine, aspartate and glutamate metabolism \\
\hline 11 & Threonic acid & $\mathrm{C}_{4} \mathrm{H}_{8} \mathrm{O}_{5}$ & 0.101 & 0.906 & 0.428 & Ascorbate and aldarate metabolism \\
\hline $12^{d}$ & L-Tryptophan & $\mathrm{C}_{11} \mathrm{H}_{12} \mathrm{~N}_{2} \mathrm{O}_{2}$ & -0.108 & 0.054 & 0.186 & Glycine, serine and threonine metabolism \\
\hline 13 & a-Lactose & $\mathrm{C}_{12} \mathrm{H}_{22} \mathrm{O}_{11}$ & -0.114 & 0.116 & 0.259 & Galactose metabolism \\
\hline $14^{\mathrm{d}}$ & L-Lactic acid & $\mathrm{C}_{3} \mathrm{H}_{6} \mathrm{O}_{3}$ & -0.129 & 0.735 & 0.263 & Propanoate metabolism \\
\hline $15^{\mathrm{d}}$ & Palmitic acid & $\mathrm{C}_{16} \mathrm{H}_{32} \mathrm{O}_{2}$ & -0.149 & 0.005 & 0.303 & Fatty acid metabolism \\
\hline 16 & Indoxyl sulfate & $\mathrm{C}_{8} \mathrm{H}_{7} \mathrm{NO}_{4} \mathrm{~S}$ & -0.164 & 0.673 & 0.583 & Unknown \\
\hline $17^{d}$ & o-Tyrosine & $\mathrm{C}_{9} \mathrm{H}_{11} \mathrm{NO}_{3}$ & -0.203 & 0.217 & 0.080 & Unknown \\
\hline 18 & p-Hydroxyphenylacetic acid & $\mathrm{C}_{9} \mathrm{H}_{8} \mathrm{O}_{3}$ & -0.122 & 0.673 & -0.120 & Tyrosine metabolism \\
\hline $19^{d}$ & Citric acid & $\mathrm{C}_{6} \mathrm{H}_{8} \mathrm{O}_{7}$ & -0.156 & 0.015 & -0.265 & Citrate cycle (TCA cycle) \\
\hline \multicolumn{7}{|l|}{ Serum: HSD vs. $H C^{a}$} \\
\hline $1^{d}$ & L-Valine & $\mathrm{C}_{5} \mathrm{H}_{11} \mathrm{NO}_{2}$ & 0.226 & 0.079 & 0.689 & Aminoacyl-tRNA biosynthesis \\
\hline $2^{d}$ & L-Leucine & $\mathrm{C}_{6} \mathrm{H}_{13} \mathrm{NO}_{2}$ & 0.161 & 0.000 & 1.120 & Aminoacyl-tRNA biosynthesis \\
\hline $3^{d}$ & L-Lysine & $\mathrm{C}_{6} \mathrm{H}_{14} \mathrm{~N}_{2} \mathrm{O}_{2}$ & 0.148 & 0.337 & 0.722 & Aminoacyl-tRNA biosynthesis \\
\hline 4 & L-Threonine & $\mathrm{C}_{4} \mathrm{H}_{9} \mathrm{NO}_{3}$ & -0.105 & 0.000 & -0.191 & Glycine, serine and threonine metabolism \\
\hline 5 & D-chiro-Inositol & $\mathrm{C}_{6} \mathrm{H}_{12} \mathrm{O}_{6}$ & -0.135 & 0.138 & -0.200 & Inositol phosphate metabolism \\
\hline $6^{d}$ & L-Methionine & $\mathrm{C}_{5} \mathrm{H}_{11} \mathrm{NO}_{2} \mathrm{~S}$ & -0.140 & 0.009 & -0.299 & Cysteine and methionine metabolism \\
\hline 7 & L-Lactic acid & $\mathrm{C}_{3} \mathrm{H}_{6} \mathrm{O}_{3}$ & -0.170 & 0.253 & -0.183 & Propanoate metabolism \\
\hline \multicolumn{7}{|l|}{ Urine: HSD vs. $H C^{a}$} \\
\hline $1^{d}$ & L-Isoleucine & $\mathrm{C}_{6} \mathrm{H}_{13} \mathrm{NO}_{2}$ & 0.161 & 0.007 & 0.681 & Valine, leucine and isoleucine degradation \\
\hline 2 & Threonic acid & $\mathrm{C}_{4} \mathrm{H}_{8} \mathrm{O}_{5}$ & 0.113 & 0.349 & 0.687 & Ascorbate and aldarate metabolism \\
\hline 3 & N-Acetyl-D-glucosamine & $\mathrm{C}_{8} \mathrm{H}_{15} \mathrm{NO}_{6}$ & 0.109 & 0.283 & 0.432 & Amino sugar and nucleotide sugar metabolism \\
\hline $4^{\mathrm{d}}$ & Stearic acid & $\mathrm{C}_{18} \mathrm{H}_{36} \mathrm{O}_{2}$ & -0.104 & 0.002 & 0.019 & Fatty acid biosynthesis \\
\hline 5 & a-Lactose & $\mathrm{C}_{12} \mathrm{H}_{22} \mathrm{O}_{11}$ & -0.116 & 0.000 & -0.126 & Galactose metabolism \\
\hline \multicolumn{7}{|l|}{ Urine: LQS vs. HSD a } \\
\hline 1 & Isobutyric acid & $\mathrm{C}_{4} \mathrm{H}_{8} \mathrm{O}_{2}$ & 0.127 & 0.304 & $1.114^{\mathrm{e}}$ & Protein digestion and absorption \\
\hline
\end{tabular}

a FC fold change, MDD major depressive disorder, HC healthy controls, LQS Liver Qi Stagnation, HSD Heart and Spleen Deficiency

b Correlation coefficients $(r)$ were obtained from OPLC-DA with a threshold of 3.010. Positive and negative coefficients respectively indicate higher and lower levels of metabolites compared to healthy controls

c $p$-values were obtained from Wilcoxon-Mann-Whitney test between TCM subtypes and healthy controls

d Metabolites were determined using standard samples

e FC value represents LQS/HSD 

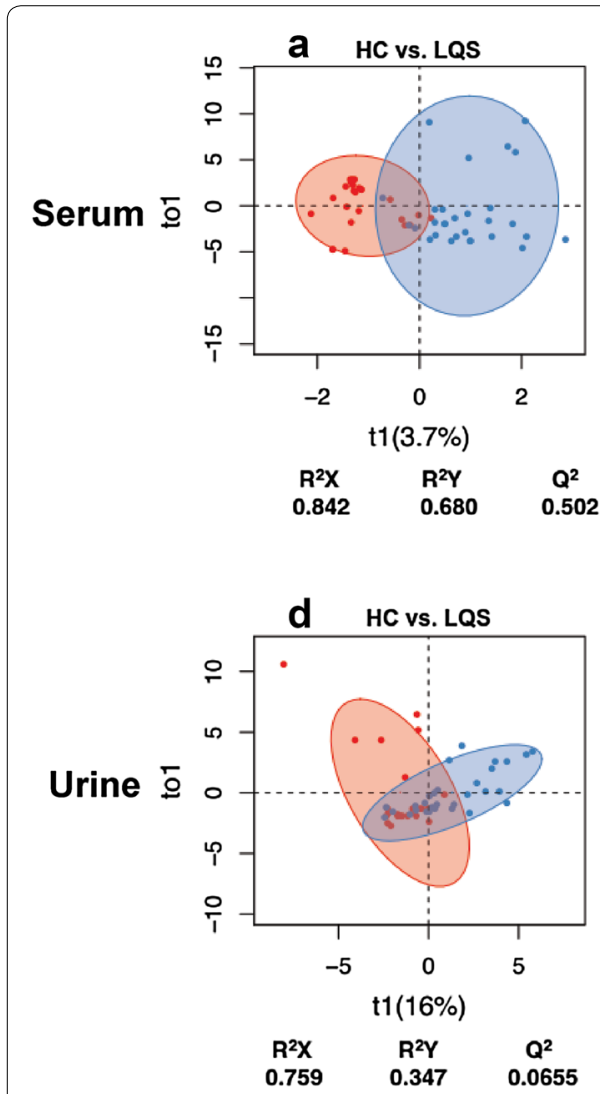
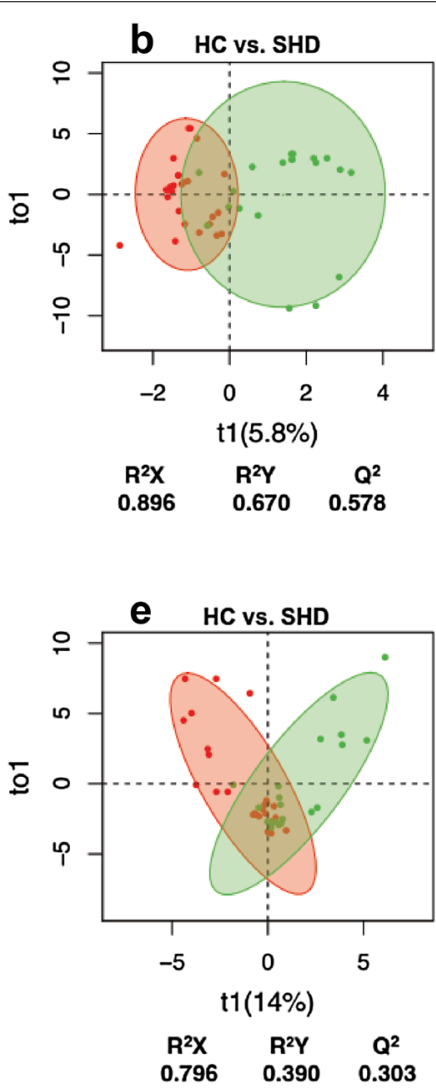
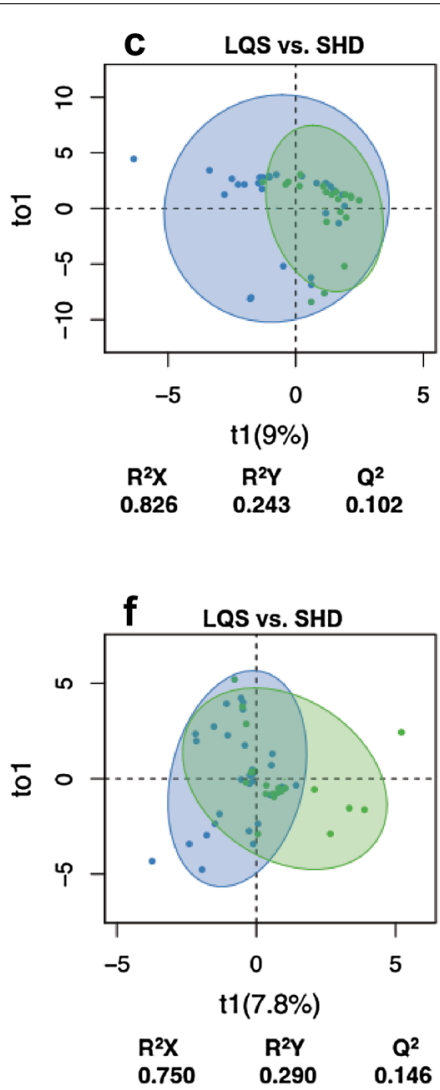

Fig. 3 Clustering analysis of serum $(\mathbf{a}, \mathbf{b}, \mathbf{c})$ and urine $(\mathbf{d}, \mathbf{e}, \mathbf{f})$ metabolomic profiles. OPLS-DA models were built in patients with Liver Qi Stagnation (LQS) and Heart and Spleen Deficiency (HSD) subtypes of MDD and healthy controls (HC). Acceptable criteria for the models were defined as all $\mathrm{R}^{2} \mathrm{X}$, $R^{2} Y$, and $Q^{2}$ values of $\geq 0.5$. Comparisons were conducted between HC and LQS (a, d), HC and HSD (b, e), and LQS and HSD (c, f)

samples but negative with the HSD subtype in serum samples. The LQS subtype had a markedly lower urinary level of isobutyric acid than the HSD subtype $(P=0.047)$.

\section{Metabolite-associated biochemical pathways}

The impact pathway analysis revealed that the differential metabolites of the LQS subtype were significantly associated with aminoacyl-tRNA biosynthesis; valine, leucine and isoleucine biosynthesis; butanoate metabolism; phenylalanine metabolism; and glycine, serine and threonine metabolism (Fig. 7a). The differential metabolites of the HSD subtype were heavily involved in aminoacyl-tRNA biosynthesis; valine, leucine and isoleucine biosynthesis; and valine, leucine and isoleucine degradation (Fig. 7b).

\section{The optimal metabolite profiles for separating TCM subtypes and healthy controls}

ROC analysis showed that the optimal metabolite profile with a combination of five serum metabolites (leucine, valine, phenylalanine, threonic acid, and glycine) well separated the LQS subtype and healthy controls with a sensitivity of 0.87 and a specificity of 0.80 (Fig. 8a). A combination of five serum metabolites (leucine, valine, threonine, methionine, and inositol) optimally dichotomized the HSD subtype and healthy controls with a sensitivity of 0.95 and a specificity of 0.88 (Fig. 8b). There were no optimal urinary metabolite profiles that could separate TCM subtypes and healthy controls at the acceptable sensitivity and specificity (data not shown).

\section{Discussion}

This study represents the first attempt to introduce TCMbased diagnostic subtypes into the modern classification of depressive disorders. It not only could improve personalized treatment of depression, but also promote the integration of TCM theoretical concepts into the modern biomedical knowledge system. The sample recruited in this study was representative of MDD patients seen in China $[7,9,11,16]$.

The purpose of this study was to explore blood and urinary metabolomic evidence that could validate TCM diagnostic classification of MDD. A total of 28 meaningful metabolites were identified as potential biomarkers for differentiating MDD and healthy subjects. These 


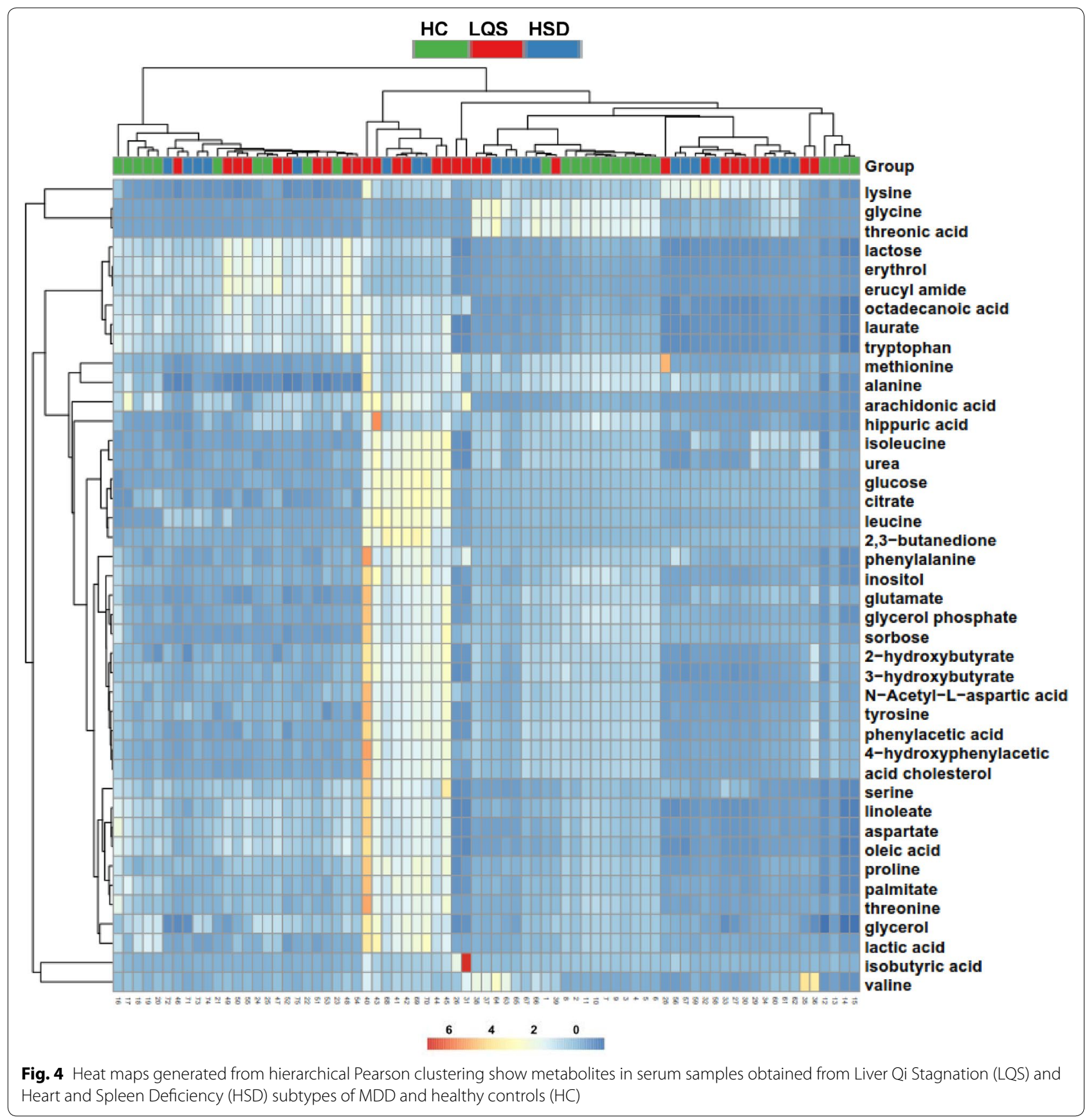

metabolites mainly included amino acid metabolites involved in the synthesis of monoamine and amino acid neurotransmitters (L-tryptophan, phenylalanine, $o$-tyrosine, glycine, 3 -hydroxybutric acid, and $\mathrm{N}$-acetylL-aspartic acid), proteinogenic branched-chain amino acids (BCAAs) (valine, leucine, and isoleucine), oxaloacetate-derived amino acids (L-lysine, L-threonine and L-methionine) which are mainly involved in the biosynthesis of proteins and energy production via gluconeogenesis, and energy metabolism-related metabolites (L-lactic acid, palmitic acid, citric acid, stearic acid, pyruvic acid, and $\alpha$-lactose) [24].

Clustering analyses revealed that the two TCM subtypes of MDD were well separated from healthy controls on serum metabolic profiles, but not on the urinary profiles. Likewise, ROC analysis displayed that the two optimal metabolite combination patterns for dichotomizing the TCM subtypes of MDD and healthy subjects 


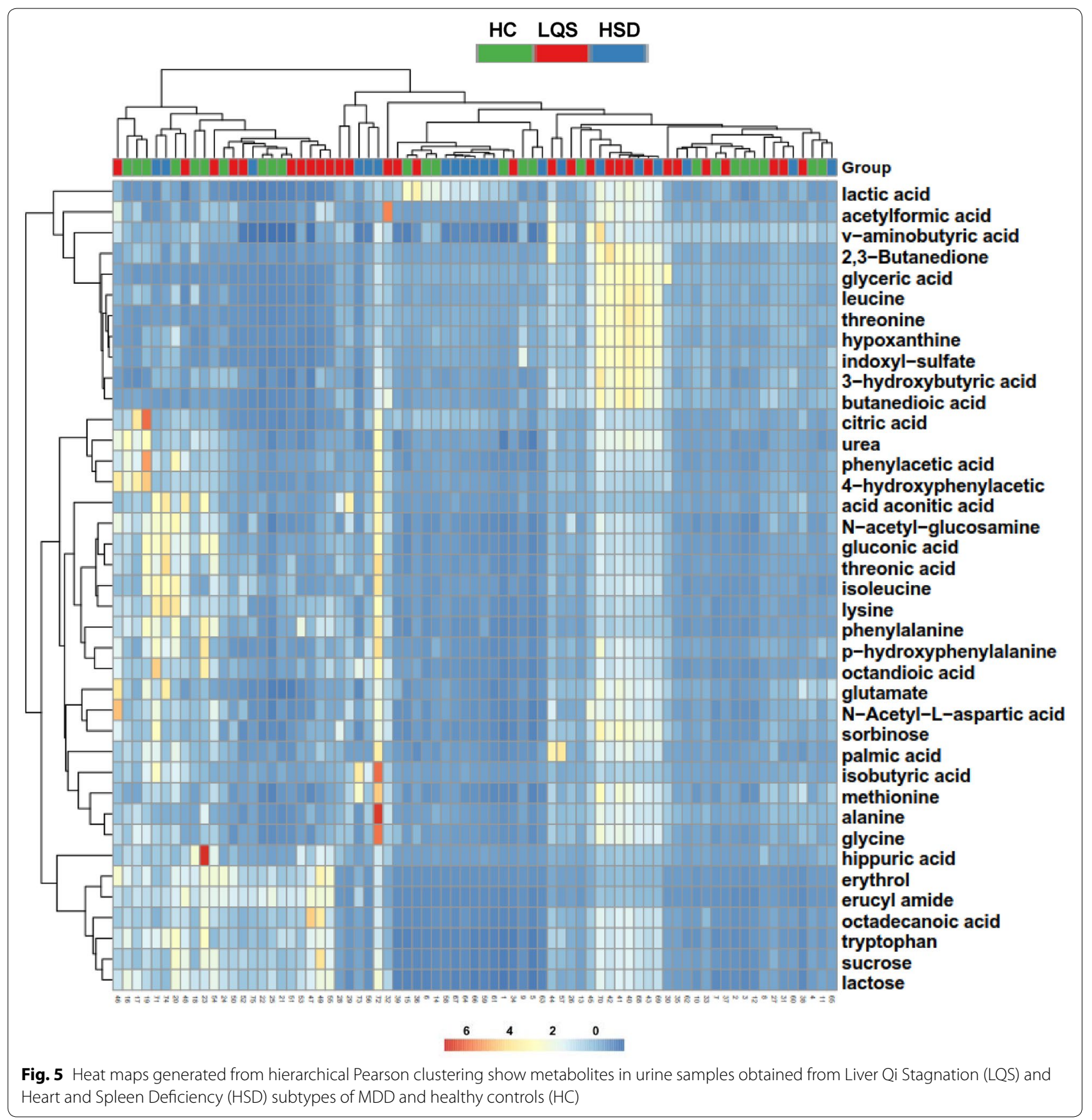

were identified from serum samples, but not from urine samples. Similar results also have been observed in previous studies that attempted to identify blood and urine metabolic biomarkers for the severity of $\operatorname{MDD}[16,20]$. Blood metabolites seem to be more sensitive than urinary metabolites in distinguishing MDD from healthy subjects. This may be largely because blood metabolites can be maintained at a relatively constant state compared to urinary metabolites [25].

Although clustering analyses showed large overlaps in serum and urinary metabolite patterns between the two TCM subtypes of MDD, the LQS subtype had strong mixed correlations with multiple amino acid metabolites involved in the biosynthesis of monoamine and 


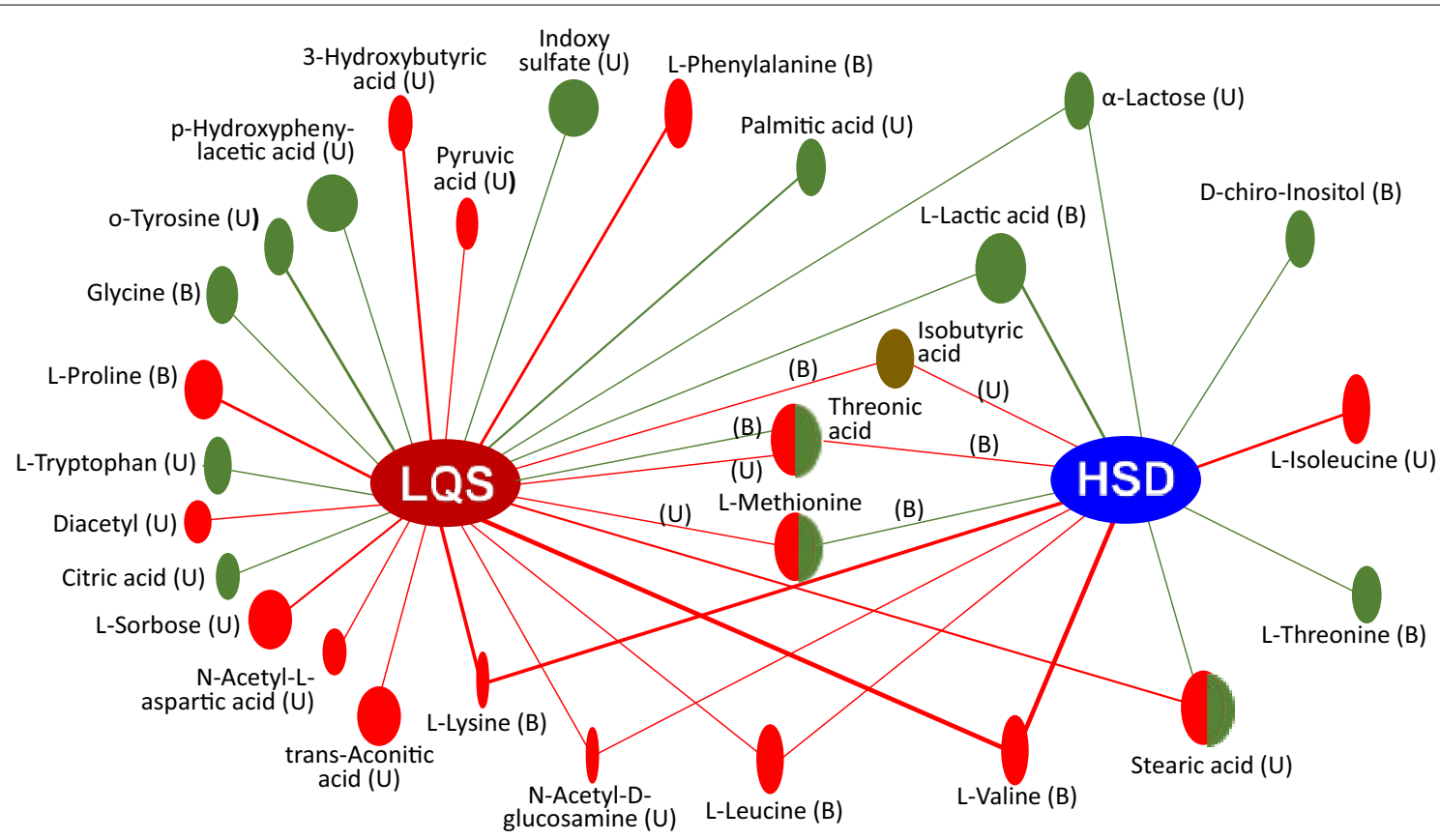

Fig. 6 Correlation network analysis of 28 differential metabolites among Liver Qi Stagnation (LQS) and Heart and Spleen Deficiency (HSD) subtypes of MDD versus healthy controls. Green and red lines with ovals indicates negative and positive correlation, respectively. The ovals with half green and half red colors indicate mixed correlations. The oval size represents $p$ value compared with healthy controls. Isobutyric acid (dark brown oral) has a significant difference between the two subtypes. $B$ blood samples, $U$ urine samples

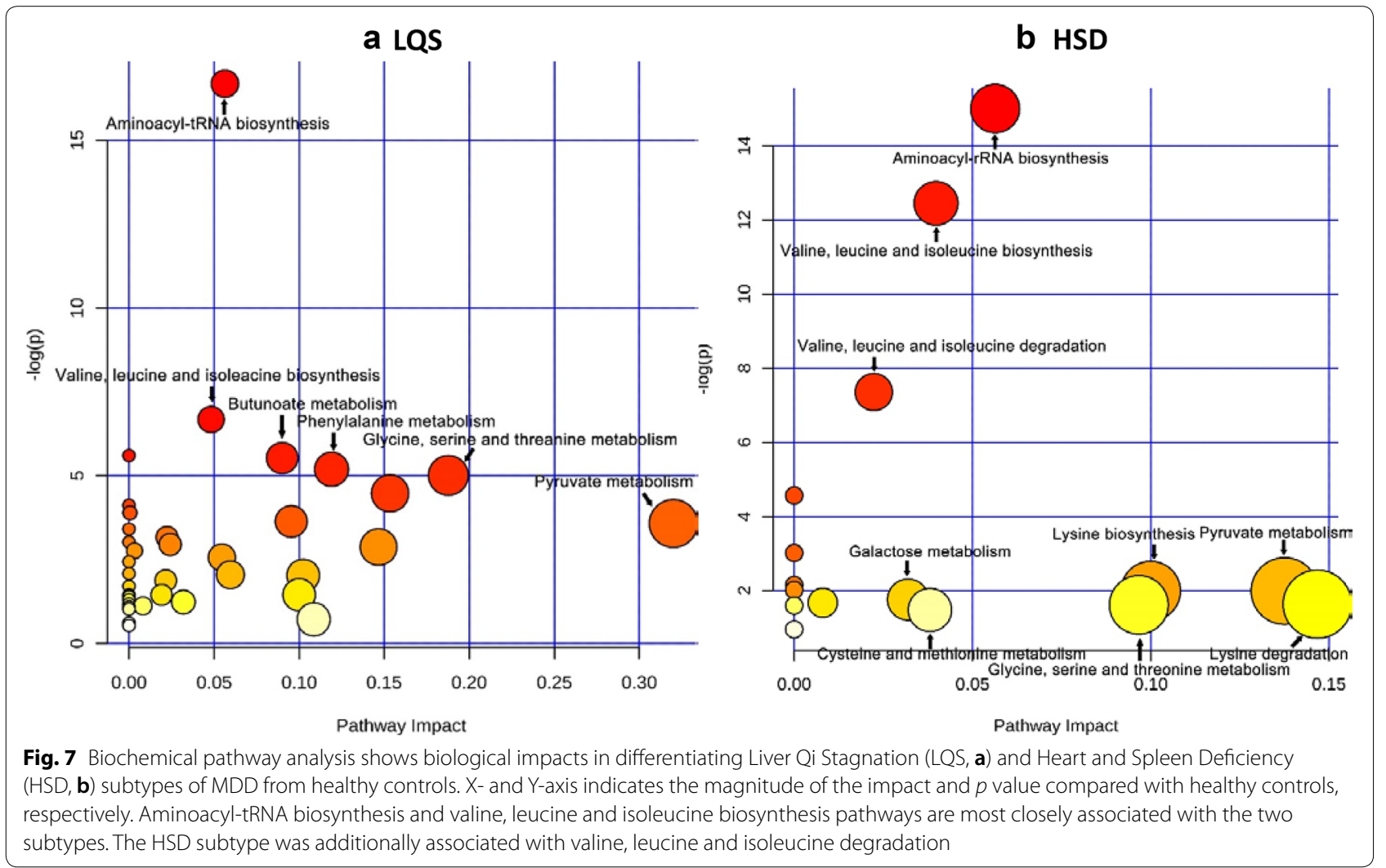



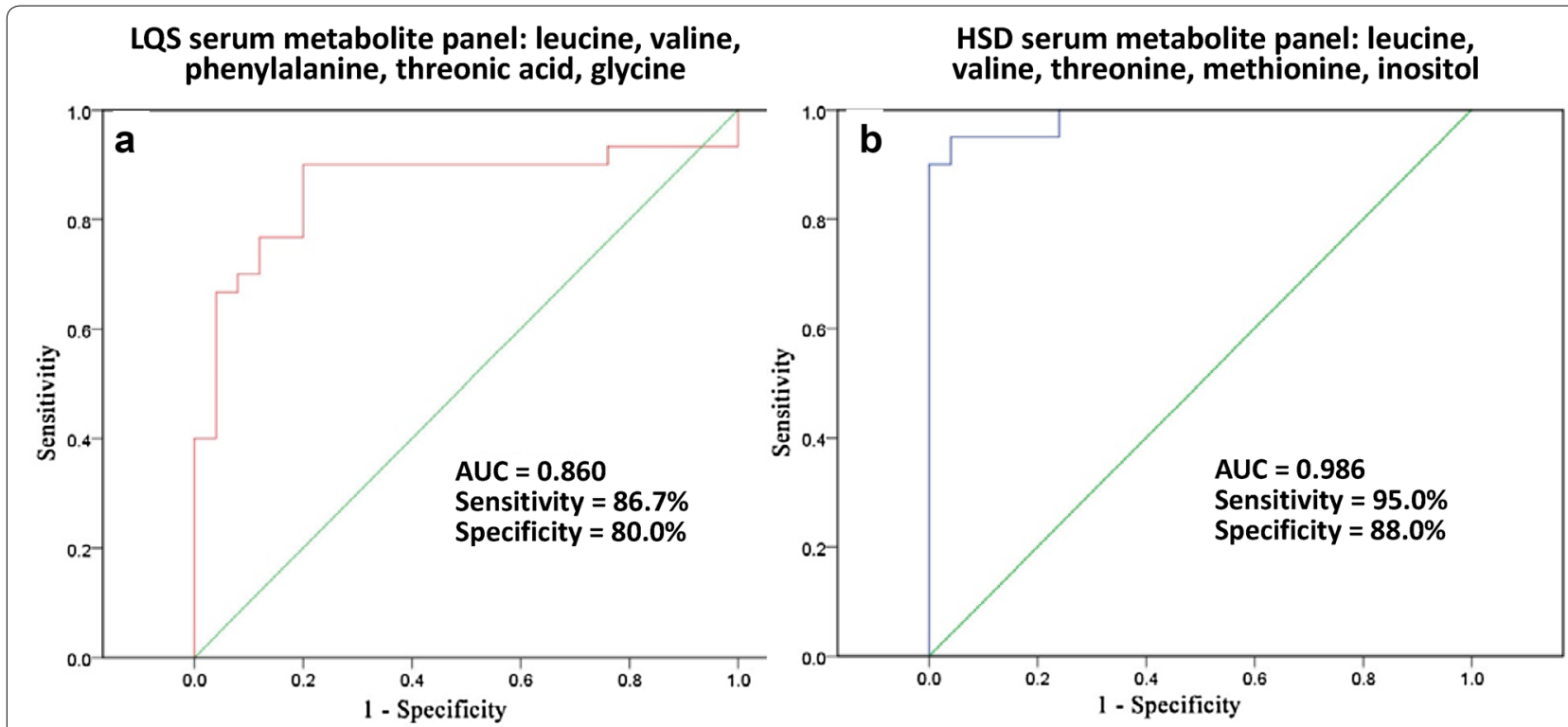

Fig. 8 Receiver operating curve (ROC) analysis show the optimal serum metabolite panels that well differentiate Liver Qi Stagnation (LQS, a) and Heart and Spleen Deficiency (HSD, b) subtype from healthy controls

amino acid neurotransmitters, including phenylalanine, 3-hydroxybutric acid, $o$-tyrosine, glycine, L-tryptophan, and $N$-acetyl-L-aspartic acid; but the HSD subtype did not. ROC analysis further indicated that phenylalanine and glycine were among the optimal metabolite combination pattern for discriminating the LQS subtype and healthy controls. A preliminary study also has observed differences in multiple brain monoamine neurotransmission among TCM subtypes of MDD [26]. According to the presence and absence of stress prior to its onset, MDD can be classified as reactive and endogenous (melancholic) subtypes [2]. Stress-associated depression has been suggested to be more closely associated with dysfunction of brain catecholamine and amino acid neurotransmitters, in particular norepinephrine, glutamate and $\gamma$-aminobutyric acid systems [27, 28]. Clinically, the LQS subtype patients often exhibit stress-related comorbid symptoms and signs, such as nervousness, irritability, agitation, and a wiry pulse [2, 10]. It thus appears that the LQS subtype may represent a subpopulation of MDD characterized by abnormal biosynthesis of monoamine and amino acid neurotransmitters and closer associations with stress-related pathophysiology.

Moreover, both subtypes had opposing correlations with serum threonic acid, serum/urine L-methionine, and urine stearic acid. Serum threonic acid and L-methionine were also among the optimal metabolite combination patterns for respectively discriminating the LQS and HSD subtype from healthy controls. Threonic acid is a metabolite of ascorbic acid (vitamin C) and methionine is an essential amino acid in humans [24]. Dietary treatment with threonic acid- and methionine-derived supplements (e.g., magnesium-Lthreonate $(\mathrm{MgT})$ and S-adenosyl-methionine (SAMe)) had the benefits in improving multiple brain functions, in particular memory, depression, and anxiety [29-31]. Stearic acid is a saturated fatty acid which plasma concentrations have been found to be abnormally altered in patients with winter episode of MDD [32] and in the superior temporal gyrus and orbitofrontal cortex of bipolar patients [33,34]. We further found that the LQS subtype had a markedly lower urinary level of isobutyric acid, a short-chain fatty acid than the HSD subtype. Plasma level of $\beta$-amino-isobutyric acid of autistic children has been shown to be significantly higher than that of healthy children [35]. These results suggest that threonic acid, methionine, stearic acid, and isobutyric acid are differentially associated with the two TCM subtypes and may be promising biomarkers for TCM classification of MDD.

However, the present study revealed that both subtypes had large overlaps in serum and urinary metabolite panels and shared several similar profiles, in particular proteinogenic BCAAs (valine and leucine) and energy metabolism-related metabolites ( $\alpha$-lactose and L-lactic 
acid), manifesting as consistent positive correlations with serum levels of valine and leucine, and negative correlations with levels of urine $\alpha$-lactose and serum L-lactic acid. Biochemical pathway analysis displayed that both subtypes had consistent and closer links with valine, leucine, and isoleucine biosynthesis and aminoacyl-tRNA biosynthesis. ROC analysis also revealed that valine and leucine were involved in the two optimal metabolite combination patterns identified for discriminating the two subtypes and healthy controls. BCAAs and energy metabolism dysfunction is related to the pathogenesis of multiple psychiatric disorders and a common comorbidity in depressive disorders $[36,37]$. The BCAAs play a key role in maintaining the supply of the neurotransmitters glutamate and $\gamma$-aminobutyric acid (GABA) [38]. Our study suggests that aberrant BCAA and energy metabolism seems to be distinct biochemical profiles in the pathophysiology of MDD and the BCAA-glutamate/ GABA metabolic cycle in the brain may be potential biomarkers and therapeutic targets for $\operatorname{MDD}[36,38,39]$.

Several limitations of this study should be noted. First, there are 12 subtypes according to TCM classification of MDD [7-11], but we only examined the two most common and opposing subtypes of MDD. Whether other subtypes also have differential profiles needs further investigation. Second, the TCM diagnostic procedure was conducted mainly based on empirical practice and subjective assessments. Objective and precise TCM diagnostic approaches should be sought. Third, the sample size was relatively small with medication-free participant and gender imbalance. This may limit the applicability of the findings of this study. Whether similar results could be achieved in first-episode and treatment-resistant depressed populations needs further determination. Finally, we did not examine the differential response of the two subtypes to antidepressant treatment. Whether metabolomic biomarkers identified are effective in predicting TCM subtype-specific treatment outcomes deserves further examination.

\section{Conclusions}

While the two subtypes are associated with aberrant BCAA and energy metabolism, the LQS subtype may represent a subpopulation of MDD characterized by abnormal biosynthesis of monoamine and amino acid neurotransmitters and closer associations with stressrelated pathophysiology. Threonic acid, methionine, stearic acid, and isobutyric acid are differentially associated with the two subtypes and may be promising biomarkers for TCM classification of MDD. This study suggests that the TCM diagnostic subtypes could serve a valid classification of MDD.

\section{Additional files}

Additional file 1. IRB approval copy.

Additional file 2. Minimum Standards of Reporting Checklist.

\begin{abstract}
Abbreviations
ANOVA: analysis of variance; BCAAs: proteinogenic branched-chain amino acids; DSM-5: Statistical Manual of Mental Disorders, Fifth Edition; GABA: Y-aminobutyric acid; GC-MS: gas chromatography-mass spectrometry; HAMD-24: 24-item Hamilton Rating Scale for Depression; HSD: Heart and Spleen Deficiency; LQS: Liver Qi Stagnation; MDD: major depressive disorder; OPLS-DA: latent structures-discriminant analysis; PCA: principal component analysis; ROC: receiver operating characteristic; TCM: traditional Chinese medicine; VIP: variable importance in the projection.
\end{abstract}

\section{Authors' contributions}

LYL and ZZJ were involved in conception and design of the study, data analysis, and preparation of the manuscript. HJZ, JBP, and WQL were responsible for metabolomic experiments and data analysis. LYLuo, CQZ, YPL, PRW, YYZ, and CYY recruited subjects, conducted clinical assessment, and collected clinical data. All authors read and approved the final manuscript.

\section{Author details}

${ }^{1}$ Department of Psychiatry, Tongde Hospital of Zhejiang Province, Hangzhou 310012, Zhejiang, China. ${ }^{2}$ Zhejiang Academy of Traditional Chinese Medicine, Hangzhou 310007, Zhejiang, China. ${ }^{3}$ Department of Internal

Chinese Medicine, Tongde Hospital of Zhejiang Province, Hangzhou 310012, Zhejiang, China. ${ }^{4}$ School of Chinese Medicine, LKS Faculty of Medicine, The University of Hong Kong, 10 Sassoon Road, Pokfulam, Hong Kong, China.

\section{Acknowledgements}

Not applicable.

\section{Competing interests}

The authors declare that they have no competing interests.

\section{Availability of data and materials}

The materials and data of this study are available to other researchers upon request.

\section{Consent for publication}

All authors have provided consent for publication in the journal of Chinese Medicine.

\section{Ethics approval and consent to participate}

This study was approved by the Medical Ethical Committee of Tongde Hospital and registered at http://www.clinicaltrials.gov (NCT02346682) before the recruitment started. All participants gave voluntary, written, informed consent before entering the study.

\section{Funding}

This study was supported by the National Natural Science Foundation of China (81403502, L.Y.L.) and Research Grant Council of Hong Kong (17115017, 17124119, Z.J.Z.).

\section{Publisher's Note}

Springer Nature remains neutral with regard to jurisdictional claims in published maps and institutional affiliations.

Received: 3 August 2018 Accepted: 18 October 2018

Published online: 25 October 2018

\section{References}

1. Kennedy SH. Core symptoms of major depressive disorder: relevance to diagnosis and treatment. Dialogues Clin Neurosci. 2008;10:271-7. 
2. Malki K, Keers R, Tosto MG, et al. The endogenous and reactive depression subtypes revisited: integrative animal and human studies implicate multiple distinct molecular mechanisms underlying major depressive disorder. BMC Med. 2014;12:73.

3. van Loo HM, de Jonge P, Romeijn JW, Kessler RC, Schoevers RA. Datadriven subtypes of major depressive disorder: a systematic review. BMC Med. 2012;10:156

4. Ilgen MA, Downing K, Zivin K, Hoggatt KJ, Kim HM, Ganoczy D, Austin $\mathrm{KL}$, McCarthy JF, Patel JM, Valenstein M. Exploratory data mining analysis identifying subgroups of patients with depression who are at high risk for suicide. J Clin Psychiatry. 2009;70:1495-500.

5. Milaneschi Y, Lamers F, Peyrot WJ, Abdellaoui A, Willemsen G, Hottenga JJ, Jansen R, Mbarek H, Dehghan A, Lu C, CHARGE inflammation working group, Boomsma DI, Penninx BW. Polygenic dissection of major depression clinical heterogeneity. Mol Psychiatry. 2016;21:516-22.

6. Cheung F. Modern TCM: enter the clinic. Nature. 2011;480:594-5.

7. Chen Z, Hu S, Zhang H, Zhang H, Zheng L, Li X. Study on criteria for commonly seen TCM syndrome of depression. JTCM. 2005;46:47-9 (Article in Chinese)

8. Guo R, Yu M, Wang JL, et al. Establishing a TCM pattern element identification scale for depression. J Beijing Univ TCM. 2015;38:561-5 (Article in Chinese).

9. Hu SY, Wang SE, Zhang CH. Relationship between symptom stratification and syndrome differentiation of traditional Chinese medicine for depressive episode. Zhong Xi Yi Jie He Xue Bao. 2011;9:933-6 (Article in Chinese).

10. China Academy of Chinese Medical Sciences and China Association for Acupuncture and Moxibustion. Evidence-based guidelines of clinical practice in Chinese medicine: acupuncture. Beijing: China Traditional Chinese Medicine Publishing House; 2011. p. 107.

11. Zhou L, Chen WK, Mei XY. Study on the characteristics of population distribution of TCM syndromes and its related factors in patients of depression. Zhongguo Zhong Xi Yi Jie He Za Zhi. 2006;26:106-9 (Article in Chinese).

12. Yeung WF, Chung KF, Zhang NL, Zhang SP, Yung KP, Chen PX, Ho YY, Identification of Chinese medicine syndromes in persistent insomnia associated with major depressive disorder: a latent tree analysis. Chin Med. 2016;11:4

13. Zhang YF, Han Y, Wang YZ, et al. Characterization of resting-state fMRIderived functional connectivity in patients with deficiency versus excess patterns of major depression. Complement Ther Med. 2015;23:7-13.

14. Fu S, Xie ZY, Yu CJ, Gao XY. Methods to establishment of serum induced syndrome of liver-depression and spleen-deficiency cell model. Zhongguo Zhong Yao Za Zhi. 2018;43:2999-3005.

15. Peng GJ, Tian JS, Gao XX, Zhou YZ, Qin XM. Research on the pathological mechanism and drug treatment mechanism of depression. Curr Neuropharmacol. 2015;13:514-23.

16. Chen JJ, Zhou CJ, Zheng P, et al. Differential urinary metabolites related with the severity of major depressive disorder. Behav Brain Res. 2017;332:280-7.

17. Ding X, Yang S, Li W, et al. The potential biomarker panels for identification of Major Depressive Disorder (MDD) patients with and without early life stress (ELS) by metabonomic analysis. PLoS ONE. 2014;9:e97479.

18. Liu $X$, Zheng $P$, Zhao $X$, et al. Discovery and validation of plasma biomarkers for major depressive disorder classification based on liquid chromatography-mass spectrometry. J Proteome Res. 2015;14:2322-30.

19. Liu Y, Yieh L, Yang T, et al. Metabolomic biosignature differentiates melancholic depressive patients from healthy controls. BMC Genomics. 2016;17:669.

20. Setoyama D, Kato TA, Hashimoto R, et al. Plasma metabolites predict severity of depression and suicidal ideation in psychiatric patients-a multicenter pilot analysis. PLoS ONE. 2016;11:e0165267.
21. Hamilton M. A rating scale for depression. J Neurol Neurosurg Psychiatry. 1960;23:56-62.

22. Ye J, Cai X, Cao P. Problems and prospects of current studies on the microecology of tongue coating. Chin Med. 2014;9:9.

23. Zheng $\mathrm{P}$, Chen JJ, Huang $\mathrm{T}$, et al. A novel urinary metabolite signature for diagnosing major depressive disorder. J Proteome Res. 2013;12:5904-11.

24. Nelson DL, Cox MM. Principles of Biochemistry. 4th ed. New York: W. H. Freeman; 2005.

25. Barrios C, Spector TD, Menni C. Blood, urine and faecal metabolite profiles in the study of adult renal disease. Arch Biochem Biophys. 2016;589:81-92.

26. Kim KN, Li YH, Xiang TY. TCM syndrome differentiation of depression and the clinical S-Et analysis. Chin J Exp Tradit Med Formulae. 2010;16:215-8.

27. Gao SF, Bao AM. Corticotropin-releasing hormone, glutamate, and Y-aminobutyric acid in depression. Neuroscientist. 2011;17:124-44.

28. Moret C, Briley M. The importance of norepinephrine in depression. Neuropsychiatr Dis Treat. 2011;7(Suppl 1):9-13

29. Huang $Y$, Huang $X$, Zhang $L$, et al. Magnesium boosts the memory restorative effect of environmental enrichment in Alzheimer's disease mice. CNS Neurosci Ther. 2018;24:70-9.

30. Sharma A, Gerbarg P, Bottiglieri T, et al. S-Adenosylmethionine (SAMe) for neuropsychiatric disorders: a clinician-oriented review of research. J Clin Psychiatry. 2017;78:e656-67.

31. Slutsky l, Abumaria N, Wu LJ, et al. Enhancement of learning and memory by elevating brain magnesium. Neuron. 2010;65:165-77.

32. Otoki Y, Hennebelle M, Levitt AJ, et al. Plasma phosphatidylethanolamine and triacylglycerol fatty acid concentrations are altered in major depressive disorder patients with seasonal pattern. Lipids. 2017;52:559-71.

33. McNamara RK, Jandacek R, Rider T, et al. Deficits in docosahexaenoic acid and associated elevations in the metabolism of arachidonic acid and saturated fatty acids in the postmortem orbitofrontal cortex of patients with bipolar disorder. Psychiatry Res. 2008;160:285-99.

34. McNamara RK, Rider T, Jandacek R, Tso P. Abnormal fatty acid pattern in the superior temporal gyrus distinguishes bipolar disorder from major depression and schizophrenia and resembles multiple sclerosis. Psychiatry Res. 2014;215:560-7.

35. Zaki MM, Abdel-Al H, Al-Sawi M. Assessment of plasma amino acid profile in autism using cation-exchange chromatography with postcolumn derivatization by ninhydrin. Turk J Med Sci. 2017;47:260-7.

36. Baranyi A, Amouzadeh-Ghadikolai O, von Lewinski D, et al. Branchedchain amino acids as new biomarkers of major depression-a novel neurobiology of mood disorder. PLoS ONE. 2016;11:e0160542.

37. Zuccoli GS, Saia-Cereda VM, Nascimento JM, Martins-de-Souza D. The energy metabolism dysfunction in psychiatric disorders postmortem brains: focus on proteomic evidence. Front Neurosci. 2017;11:493.

38. Cole JT, Sweatt AJ, Hutson SM. Expression of mitochondrial branchedchain aminotransferase and a-keto-acid dehydrogenase in rat brain: implications for neurotransmitter metabolism. Front Neuroanat. 2012;6:18.

39. Mathews DC, Henter ID, Zarate CA. Targeting the glutamatergic system to treat major depressive disorder: rationale and progress to date. Drugs. 2012;72:1313-33

Ready to submit your research? Choose BMC and benefit from:

- fast, convenient online submission

- thorough peer review by experienced researchers in your field

- rapid publication on acceptance

- support for research data, including large and complex data types

- gold Open Access which fosters wider collaboration and increased citations

- maximum visibility for your research: over $100 \mathrm{M}$ website views per year

At BMC, research is always in progress.

Learn more biomedcentral.com/submissions 